\title{
Effect of Airflow Rate on Vibration Response Imaging in Normal Lungs
}

\author{
Meirav Yosef ${ }^{1}$, Ruben Langer ${ }^{1}$, Shaul Lev ${ }^{2}$ and Yael A. Glickman ${ }^{*}$ \\ ${ }^{I}$ Deep Breeze, Ltd., 2 Hailan St., P.O. Box 140, Or-Akiva, 30600, Israel \\ ${ }^{2}$ Department of General Intensive Care, Rabin Medical Center, Beilinson Campus, 39 Jabotinski St., Petach Tikva, \\ 49100, Israel
}

\begin{abstract}
Background: Evaluating the effect of airflow rate on amplitude of lung sound energy and regional distribution of lung sounds may assist in the interpretation of computerized acoustic measurements.

Objectives: The aim of this study was to assess the effect of airflow rate on Vibration Response Imaging (VRI) measurement in healthy lungs.

Methods: Lung sounds were recorded from 20 healthy adults in the frequency range of $150-250 \mathrm{~Hz}$ using 40 piezoelectric sensors positioned on the posterior chest wall. During the recordings, subjects were breathing at airflow rates ranging between 0.3 and $1.7 \mathrm{~L} / \mathrm{s}$. Online visual feedback was provided using a pneumotach mouthpiece.

Results: Amplitude of lung sound energy significantly increased with increasing airflow rate $(\mathrm{p}<0.00001$, Friedman test). A strong relationship $\left(\mathrm{R}^{2}=0.95\right)$ was obtained between amplitude of lung sound energy at peak inspiration and airflow rate raised to the third power. This correlation did not significantly affect normalized lung sound distribution maps at peak inspiration, especially when airflow was higher than $1.0 \mathrm{~L} / \mathrm{s}$. Acoustic maps obtained at airflow rates below $0.7 \mathrm{~L} / \mathrm{s}$ differed from those recorded above $1.0 \mathrm{~L} / \mathrm{s}(\mathrm{p}<0.05$, Wilcoxon matched-paired signed-ranks test).
\end{abstract}

Conclusion: These findings may be of importance when comparing healthy and diseased lungs or when monitoring changes in lung sounds during treatment follow-up.

Keywords: Lung sounds, airflow rate, VRI, regional ventilation distribution, acoustic energy, acoustic map.

\section{INTRODUCTION}

Mapping of lung sound distribution in the human thorax is a growing field of interest. Dosani and Kraman described a display technique by which contour maps of lung sound intensity were constructed in healthy subjects [1]. Later, Kompis and colleagues depicted a novel method for acoustic imaging of the human respiratory system using a series of multisensors simultaneously recording thoracic sounds from the chest wall. A spatial representation of the recorded sounds revealed structural and functional properties of the respiratory system [2]. Acoustic thoracic imaging was further explored by CharlestonVillalobos et al. when comparing different interpolation techniques while positioning a $5 \times 5$ microphone array on the anterior and the posterior thoracic surfaces and recording lung sounds [3]. The same investigators also examined acoustic maps resulting from glottal sound transmission through the bronchiallung-thoracic system [4].

Recently, Dellinger and colleagues described the dynamic visualization of lung sounds with a vibration response imaging (VRI) monitoring system [5]. While breathing flow, volume and timing were not documented, standardization of breathing intensity was obtained from feedback provided by the breathing intensity bar of the

*Address correspondence to this author at the Deep Breeze, Ltd., 2 Hailan St., P.O. Box 140, Or-Akiva, 30600, Israel; Tel: +972 4 6266650, Ext. \#227; Fax:+972 4 6266653; E-mail: yael.glickman@deepbreeze.com instrument. Furthermore, the same authors reported the effect of tidal volume/airflow on sound energy in four healthy volunteers while increasing volume in the range of 0.3 to $1.5 \mathrm{~L}$ at constant respiratory rate. A linear relationship was described with coefficient of determination $\mathrm{R}^{2}$ values ranging from 0.74 to 0.82 [6]. In this same article, analytical methods were introduced to assess changes in amplitude of lung sound energy (or vibration intensity) and in normalized lung acoustic maps (or images) when changing mode of mechanical ventilation in intensive care patients. A similar approach was recently used to assess changes in regional distribution of lung sounds as a function of positive endexpiratory pressure (PEEP) [7]. Vena and colleagues also reported the acoustic effects of PEEP on normal lung sounds [8].

The aim of the present study was to evaluate the effect of airflow rate on lung sound measurement as obtained with VRI in normal lungs. Repeatability of the measurement was also evaluated.

\section{MATERIALS AND METHODOLOGY}

\section{Study Design}

Healthy adults without a history of chronic pulmonary disease and with healthy breath sounds (i.e. no adventitious lung sounds), were enrolled in this study after providing informed consent. Deep Breeze Ltd. has received approval from the ethical committee to conduct studies in healthy 
volunteers. Lung sounds from each subject were recorded five times with an approximately 3-minute rest between recordings. Subjects were instructed to maintain a constant respiratory rate of 20 breaths per minute while average inspiratory flow was adjusted from 0.3 to $1.7 \mathrm{~L} / \mathrm{s}$ (tidal volume between 0.5 and $2.5 \mathrm{~L}$ ) using a pneumotach mouthpiece. Each recording lasted 20 seconds.

\section{Recording Procedure}

A schema of the apparatus is provided in Fig. (1). The acoustic measurements were performed using a computerbased lung sound monitoring device (VRI ${ }^{\mathrm{TM}}$ System, Deep Breeze ${ }^{\mathrm{TM}}$, Or-Akiva, Israel) [9]. Subjects were seated in a quiet environment during the recordings. Lung sounds were measured using two arrays of 7 rows $\times 3$ columns of piezoelectric contact sensors or microphones similar to those used in digital stethoscopes (Meditron ASA, Oslo, Norway). Positioning of the arrays was dictated by anatomical landmarks such as spine and scapula. A low vacuum computer-controlled method was used to attach the arrays uniformly on the posterior chest wall of the exposed back.

Subjects were asked to breathe through a pneumotach mouthpiece connected to a KoKo Pneumotach Spirometer (Pulmonary Data Services, Louisville, CO) with customizable software. A real-time display of the delivered volume as a function of time was presented on a computer screen. After attachment of the piezoelectric contact sensors and initiation of the recording procedure, subjects were instructed to hold their breath for three seconds and then to breathe into the pneumotach mouthpiece. This was performed in order to synchronize the 20 -second lung sound recording with the 15 -second pneumotach signal. During the recordings, the subjects were provided real-time graphic feedback.

\section{Measurement Output}

Acoustic signals were stored digitally on the system for later review and analysis. A 64 multi-channel analog to digital converter was used to filter and amplify the sensor signals, acquired at a 16-bit acquisition level and a sampling rate of 19.2 $\mathrm{kHz}$. A band-pass filter between 150 and $250 \mathrm{~Hz}$ was applied to remove heart and muscle sounds; median filtering was performed to suppress impulse noise, and truncation of samples above an automatically determined signal-to-noise threshold was carried out. Lung sound energy was obtained following down-sampling and a total of 118 data points were collected during the 20 second recording. Each point represented 0.17 seconds of sound energy expressed in arbitrary units (AU). Sound energy was synchronized with the respiratory volumes obtained from the pneumotach spirometer hardware. The onset of inspiration, for both the sound energy and volume graphs, was detected automatically and then confirmed by visual inspection. Onset of the first complete respiratory cycle after breath hold was used to synchronize both waveforms. The analysis was performed on a representative data point corresponding to peak-inspiratory flow.

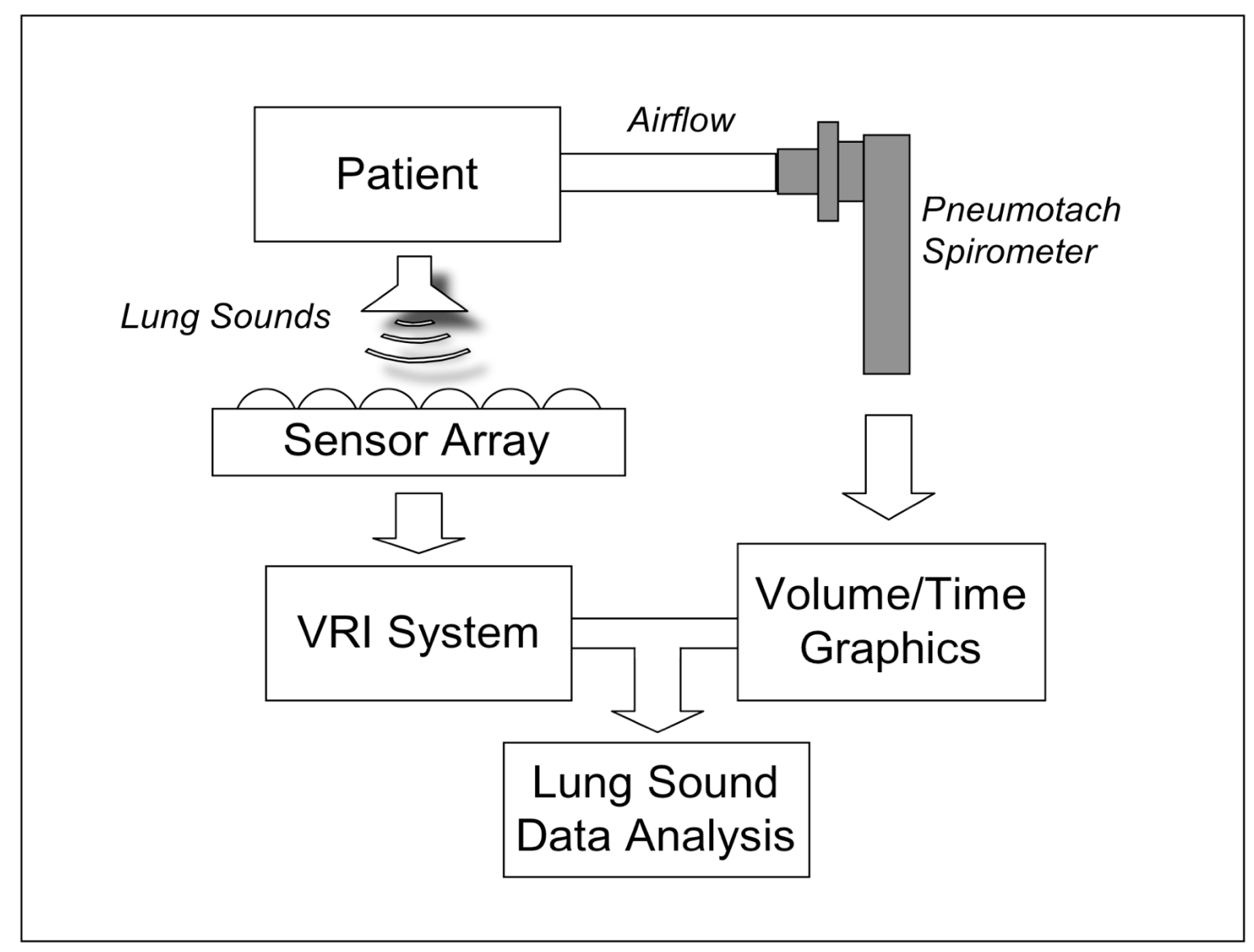

Fig. (1). Schematic diagram describing the elements of the system: patient breathing through a pneumotach mouthpiece connected to an external computer with graphic display. Lung sounds are recorded by a sensor array connected to the VRI system and acoustic data are combined with spirometer measurements for analysis. 
Data analysis was performed on amplitude of lung sound energy as well as on regional distribution of lung sounds. The absolute amplitude of sound energy, which was not affected by normalization, was obtained for each sensor and the sum of sound energy in the right and left lungs, as well as the total sound energy in both lungs, were computed off line at peak inspiration. Normalization was applied to a set of three data points around peak-inspiration. A normalized regional lung sound distribution map was generated within this range. Areas with the highest sound energy were represented as black in a gray-level scale and areas with the lowest sound energy as light gray or white. Resulting normalized acoustic maps were quantified and presented as the percentage of weighted pixels in three lung areas: upper or apical (two upper rows of sensors), middle (three middle rows of sensors) and lower or diaphragmatic (two lower rows of sensors), summing to a total of $100 \%$.

\section{Statistical Analysis}

Data are presented using descriptive statistics as mean \pm standard deviation (SD). Wilcoxon's signed-ranks test was used for paired data, Mann-Whitney U-test for unpaired data, one-way ANOVA to analyze variance between groups and Friedman test for two-way repeated measures analysis of variance by ranks. Analyses were performed using statistics software (IFA Services Statistics, Amsterdam, Holland). Coefficients of determination $\mathrm{R}^{2}$ and normalized root mean square error (NRMSE) were obtained using Microsoft ${ }^{\circledR}$ Office Excel 2003 (Microsoft Corporation, Redmond, WA). A p-value lower than 0.05 was considered significant.

\section{RESULTS}

A total of hundred (100) lung sound recordings were performed on 20 healthy adults (19 males, 1 female; age 33 \pm 6 years). Mean weight was $77 \pm 9 \mathrm{~kg}$, mean height $176 \pm 5$ $\mathrm{cm}$ and mean body mass index (BMI) $25 \pm 2$. Mean amplitude of lung sound energy recorded from all the sensors at peak inspiration increased with airflow rate as summarized in Fig. (2). Differences between recordings at $0.3,0.7,1.0,1.3$ and $1.7 \mathrm{~L} / \mathrm{s}$ were highly significant as revealed by Friedman test $(p<0.00001)$. The result of a regression analysis conducted on the cube root of mean sound energy is displayed in Fig. (3). As shown in this figure, the data was linear with a coefficient of determination $\mathrm{R}^{2}$ equal to 0.95. Furthermore, as shown in Fig. (4), a strong correlation between the actual sound energy and the predicted values obtained from the linear regression raised to the third power was obtained $\left(\mathrm{R}^{2}=0.98\right)$. The normalized root mean square error (NRMSE) between actual and predicted values was relatively small (8\%). This finding confirms a strong correlation between sound energy and the third power of airflow rate in healthy lungs during inspiration. Furthermore, mean sound energy from the left lung was 25$50 \%$ higher than from the right lung (Wilcoxon matchedpairs signed-ranks, $\mathrm{p}<0.05$ ) as illustrated in Fig. (5). Mean sound energy was also analyzed according to age, height, weight and BMI. Median for each parameter was used as cut-off. In average, sound energy was increased in younger and shorter subjects but this increase was not statistically significant in our data set (Mann-Whitney U-test).

Normalized lung sound energy maps at peak inspiration were obtained for each subject at each level of airflow. Examples of these maps are provided in Fig. (6). As shown in this figure, only minimal visual changes were detectable in normalized maps recorded between 0.3 and $1.7 \mathrm{~L} / \mathrm{s}$. Regional distributions of average weighted pixels from each map were compared across airflow rates. As revealed in Table 1, the proportion of sound energy in the right and left lungs was not altered by changes in airflow rate. Moreover, no significant differences in regional distribution were encountered between maps obtained at $1.0,1.3$ and $1.7 \mathrm{~L} / \mathrm{s}$.

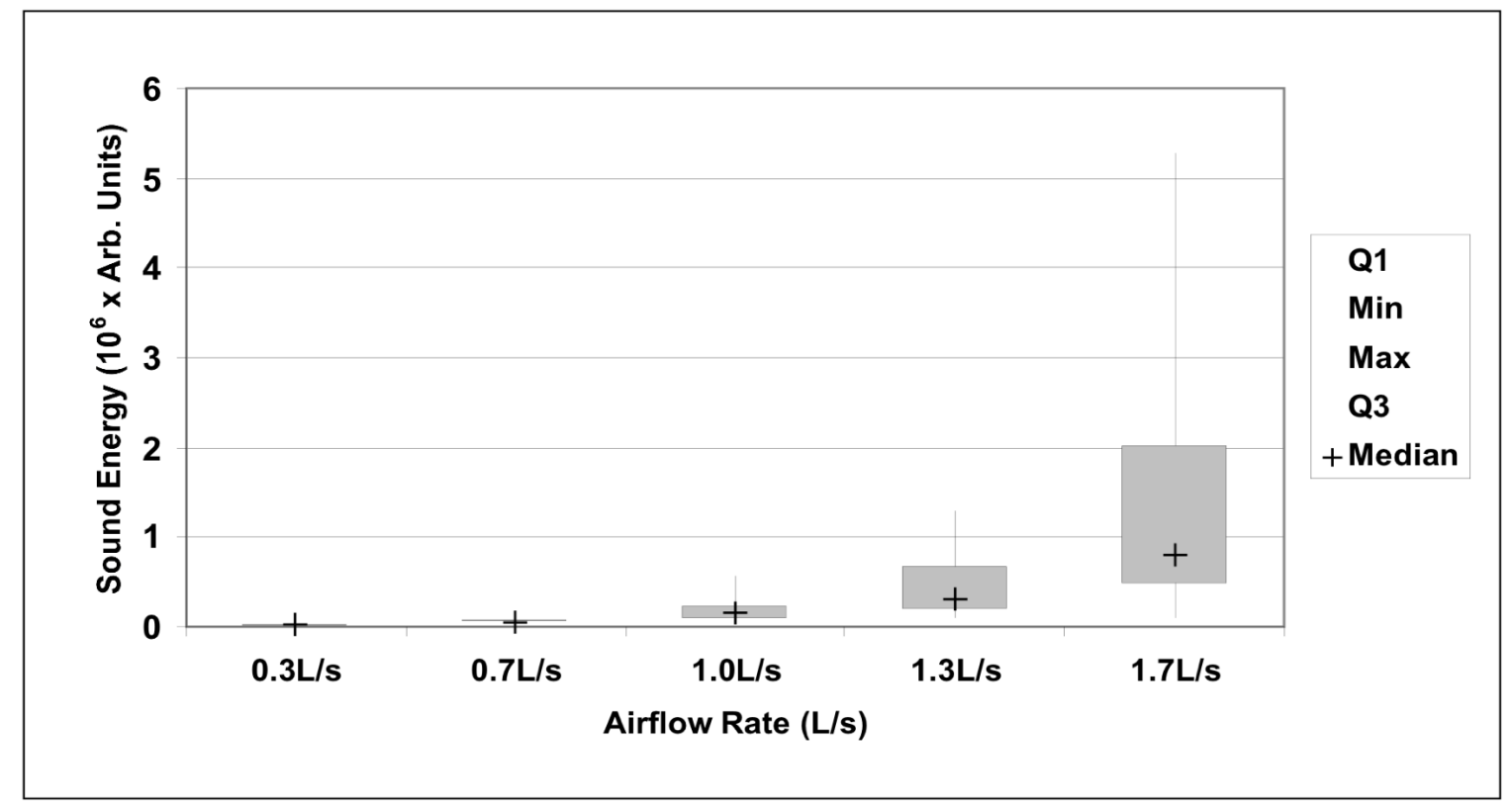

Fig. (2). Box plot representation of sound energy as a function of airflow rate $(n=20)$. Boxes indicate $25 \%$ and $75 \%$ percentiles, with the median sign inside. Error bars represent minimum and maximum values. Differences between each groups was highly significant $(\mathrm{p}<0.00001$, Wilcoxon's signed-ranks test). 


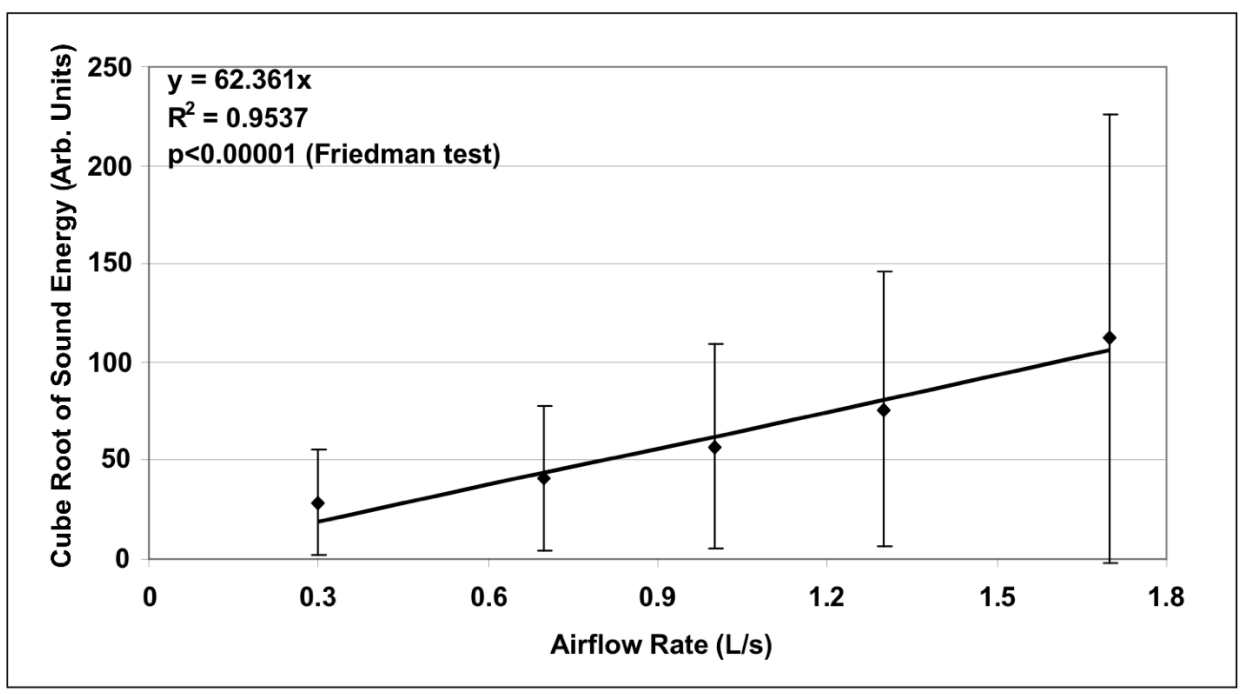

Fig. (3). Mean \pm standard deviation of the cube root of sound energy as a function of airflow rate $(n=20)$. Linear fit is represented on chart together with corresponding equation. The coefficient of determination $\mathrm{R}^{2}$ was equal to 0.95 .

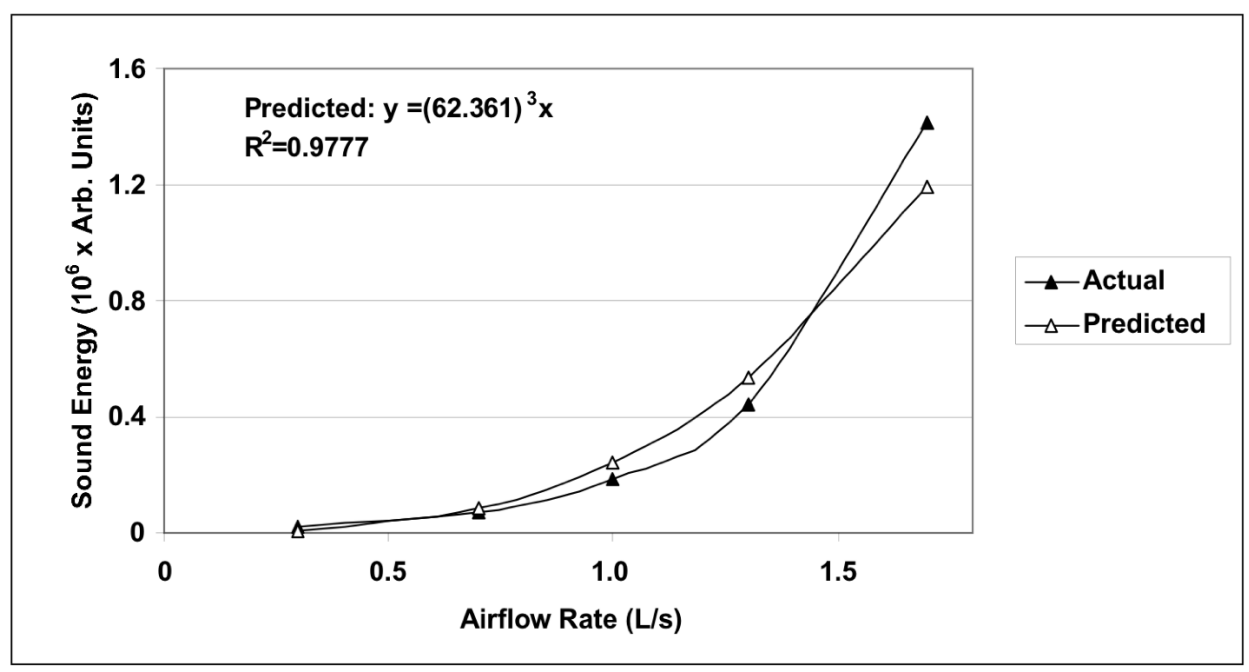

Fig. (4). Actual mean sound energy compared to predicted values obtained from the linear regression raised to the third power. The coefficient of determination $\mathrm{R}^{2}$ was equal to 0.98 . The normalized root mean square error (NRMSE) between actual and predicted values was $8 \%$.

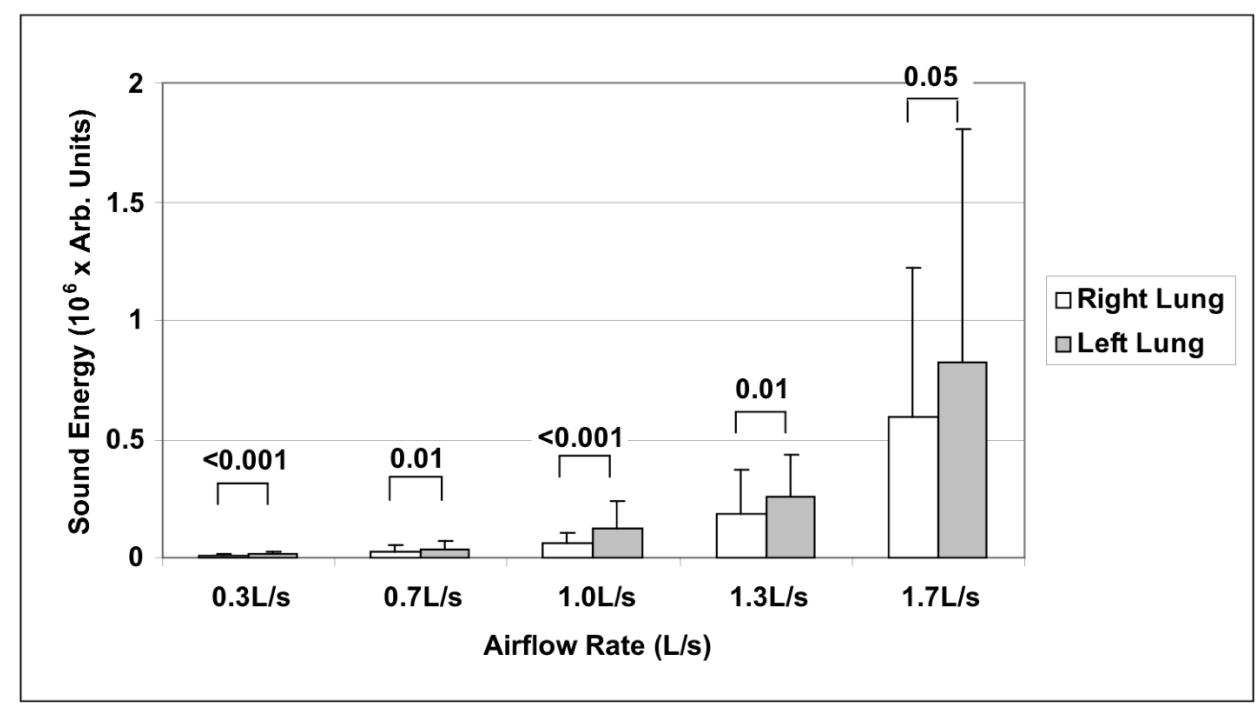

Fig. (5). Mean \pm standard deviation of sound energy in right and left lungs as a function of airflow rate ( $n=20)$. Significant $p$-values are indicated (Wilcoxon matched-pairs signed-ranks test). 


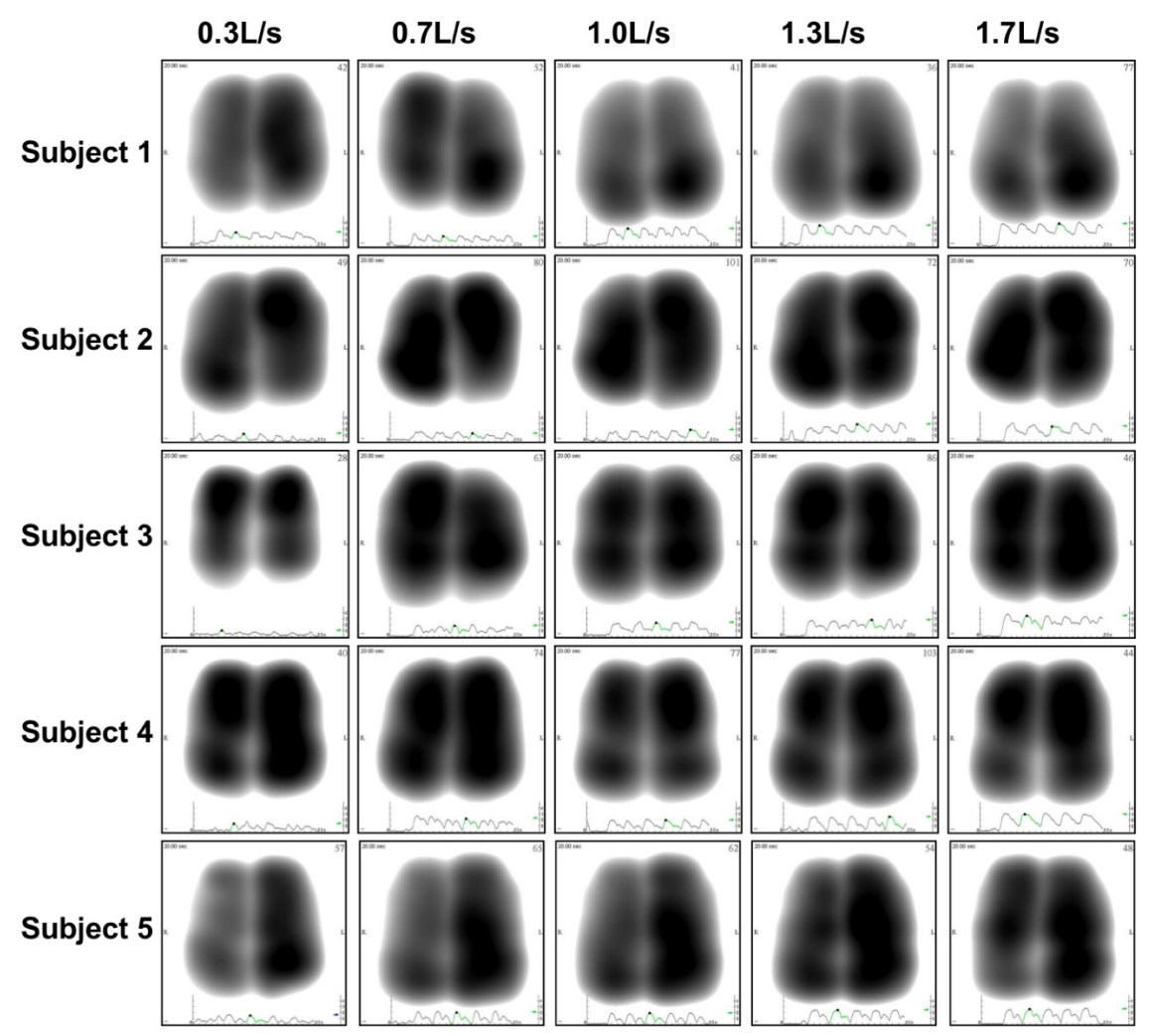

Fig. (6). Representative normalized acoustic maps at peak inspiration obtained from 5 individuals recorded at five levels of airflow rates varying from 0.3 to $1.7 \mathrm{~L} / \mathrm{s}$.

However, significant differences were encountered in the apical and diaphragmatic lung areas between lung sound distribution maps recorded at airflow rate below and above 1.0L/s (Wilcoxon Matched-Pairs Signed-Ranks).

Finally, the repeatability of the measurement was assessed in three repeated respiratory cycles for each patient at each flow rate. Repeatability was performed by comparing the distribution of sound energy in each of the six lung regions, as well as in total left and right lungs. No significant difference was encountered between repeated measurements (one-way ANOVA for repeated measures).

\section{DISCUSSION}

Although there is a general consensus that an increase of respiratory flow rate induces louder breath sounds $[10,11]$, the exact nature of the relationship between airflow and lung acoustic in normal subjects has not been firmly established [12]. A linear relationship has been reported by Leblanc [13], Kraman [14] and Hossain [15], a quadratic relationship has been described by Shykoff [16] and a third power by Olson $[17,18]$. Other relationships have been proposed [19-23] and Nakano and colleagues investigated the most suitable frequency band to analyze regional ventilation. Correlation was best at frequency bands from $150 \mathrm{~Hz}$ to $850 \mathrm{~Hz}$ with correlation coefficients exceeding 0.8 [24]. The disparity of the results reveals high variability due to method of measurement and data analysis.

In healthy lung, the total cross sectional area of the airways increases rapidly along the bronchial tree. This causes the gas flowing in the airways to decelerate while

Table 1. Mean \pm Standard Deviation of the Percentage of Weighted Pixel Counts in Left and Right Lungs as Well as in Upper (Apical), Middle and Lower (Diaphragmatic) Lung Segments at Airflow Rates Ranging Between 0.3 and $1.7 \mathrm{~L} / \mathrm{s}$. Significant Differences Encountered Between Lower and Higher Flow Ranges are Reported (Wilcoxon Matched-Pairs Signed-Ranks Tests, $p<0.05)$. NS Means No Significant Differences

\begin{tabular}{|c|c|c|c|c|c|c|}
\hline & \multicolumn{2}{|c|}{ Lower Flow Range } & \multicolumn{3}{|c|}{ Higher Flow Range } & \multirow{2}{*}{$\begin{array}{c}\text { Lower } v \text { s Higher Flov } \\
\text { p Value }\end{array}$} \\
\hline & $0.3 \mathrm{~L} / \mathrm{s}$ & $0.7 \mathrm{~L} / \mathrm{s}$ & $1.0 \mathrm{~L} / \mathrm{s}$ & $1.3 \mathrm{~L} / \mathrm{s}$ & $1.7 \mathrm{~L} / \mathrm{s}$ & \\
\hline Total Left & $58 \pm 7 \%$ & $55 \pm 7 \%$ & $57 \pm 7 \%$ & $57 \pm 8 \%$ & $54 \pm 7 \%$ & NS \\
\hline Total Right & $42 \pm 7 \%$ & $45 \pm 7 \%$ & $43 \pm 7 \%$ & $43 \pm 7 \%$ & $46 \pm 7 \%$ & NS \\
\hline Upper & $30 \pm 6 \%$ & $29 \pm 7 \%$ & $26 \pm 9 \%$ & $24 \pm 8 \%$ & $25 \pm 6 \%$ & $<0.05$ \\
\hline Middle & $33 \pm 3 \%$ & $34 \pm 4 \%$ & $32 \pm 4 \%$ & $33 \pm 5 \%$ & $32 \pm 4 \%$ & NS \\
\hline Lower & $37 \pm 8 \%$ & $37 \pm 9 \%$ & $42 \pm 11 \%$ & $44 \pm 11 \%$ & $42 \pm 8 \%$ & $<0.05$ \\
\hline
\end{tabular}


maintaining flow. This deceleration is enabled through the turbulence occurring in the multiple tree bifurcations as well as the continuous friction with the inner surface of the airways. It is within reason to assume that in healthy lung, the gradual dissipation of the flow's kinetic energy along the bronchial tree is a prime source of lung sounds that are associated with flow. In this context, total initial kinetic energy of the flow (when entering the trachea) is expected to correlate with total sound energy. However, this description only holds when the decay of sound within the parenchyma can be averaged out between all possible pathways, and when sound energy is integrated over the time required to travel along the tree. Under theses terms, the kinetic energy associated with flow can be approximated via a third order function of flow rate as experimentally observed in our study. This finding is also compatible with classical principles related to the power content of free unobstructed air moving at a constant speed in a cylindrical column [25].

Despite the strong relationship between amplitude of lung sound energy, as measured with the VRI, and airflow rate raised to the third power, the relative regional acoustic distribution, as accounted by the normalized acoustic map and related pixel count assessment, was not affected by airflow in the $1.0-1.7 \mathrm{~L} / \mathrm{s}$ range. However, an increase in regional distribution of lung sounds was observed in the more peripheral lung areas when increasing flow from 0.3$0.7 \mathrm{~L} / \mathrm{s}$ to $1.0-1.7 \mathrm{~L} / \mathrm{s}$. These findings are compatible with earlier studies describing the regional distribution of inspired gas and the effect of inspiratory flow rate on this distribution [26-28]. Milic-Emili et al. studied the expansion of different regions of the lungs at various lung volumes in seated normal men using radioactive gas. At low inspired volumes, changes were greater in the upper than in the lower lung regions, whereas the opposite was true at higher lung volumes. Ploy-Song-Sang et al. confirmed these results in a study reporting the distribution of regional ventilation measured by breath sounds in normal subjects. The authors established that ventilation to lower portions of the lung increased as lung volume increased [11]. As confirmed by our study, a certain rate is necessary in order for the flow to reach more peripheral lung areas and for lung sounds to be detectable in these areas. Once airflow is distributed in these more distal segments, changes in normalized lung sound distribution maps are less significant. In fact, the effect of normalization on the acoustic map appears to reduce the effect of changes in flow rate that are not producing changes in airflow regional distribution. This finding supports previous observation that the increase of lung sound distribution in diaphragmatic lung areas when changing mode of mechanical ventilation from volume control to pressure support is due to increase in airflow re-distribution to the diaphragmatic lung segments rather than to changes in ventilator settings [6]. Additional data points in the 0.7$1.0 \mathrm{~L} / \mathrm{s}$ airflow range may have been useful in order to further investigate this aspect.

Another interesting finding of our study was that, at each level of flow rate, lung sounds recorded in the left lung were significantly higher than in the right lung. This intrinsic right/left acoustic asymmetry has been previously reported during tidal breathing [29] and was explained by the effect of mediastinal structures and airway geometry on airflow turbulence $[1,30,31]$, particularly due to the curvature of the left bronchus. Our study further validates these findings at different levels of flow rate. Moreover, the lack of significant difference related to variables such as age, height, weight and BMI may be explained by the relatively high homogeneity of the enrolled population. Finally, the repeatability of the VRI measurement obtained in our study was comparable with previous reports in healthy individuals [29] as well as mechanically ventilated patients [7].

One of the limitations of our study is the elevated standard deviations detected in all the analyses related to amplitude of sound energy. This variability, also observed in earlier studies [6], may be explained by the algorithm used to calculate lung sound energy as the sum of squared amplitudes in a 0.17 second window. Variability of measurements could also be related to lung parenchymal sound absorption which may differ for each individual. Another limitation includes the lack of enrollment of female patients compared to male patients due to the measurement procedure requiring exposing the bare back. Since genderrelated differences in lung sounds have been reported [32], it could have been interesting to further study the impact of gender in the airflow-sound relationship. Finally, sound filtering to a band-pass of $150-250 \mathrm{~Hz}$ may have reduced the information as lung sound characteristics are contained in other frequency bands, especially above $250 \mathrm{~Hz}$.

Despite these limitations, the results obtained in this study are of particular interest since they can be used in future development of dedicated algorithms based on difference between predicted regional lung sound energies (calculated based on applied flow) and recorded energy values in diseased lungs. This could aid in the diagnosis of sound-increasing pathologies (such as consolidations) or sound-decreasing pathologies (such as atelectasis). Furthermore, the ability to differentiate between the functionality of two lungs separately could be useful in pretransplant state [33]. Comparison between normal and diseased lungs [34-37] should be further investigated in the perspective of these findings, particularly in patients with chronic obstructive pulmonary disease or asthma [38-40].

\section{CONCLUSION}

As demonstrated in this study, the effect of flow rate on normalized acoustic maps appeared to be minimal when not connected with changes in regional distribution of airflow. However, a certain level of flow rate may be necessary in order to standardize normalized acoustic maps obtained with VRI. Furthermore, the correlation between amplitude of lung sound energy and airflow raised to the third power may be of particular clinical value when predicting the expected (normal) sound energy measured from each lung region and comparing it to the actual measurement. This may be of importance when evaluating asymmetric sound distribution or when assessing treatment efficiency. This should be further investigated in diseased lungs as well as on mechanically ventilated patients.

\section{CONFLICT OF INTEREST}

MY and RL were employees of Deep Breeze, Ltd. at the time of the study. SL has a consultant agreement that includes honoraria and stock options (no current monetary value) with Deep Breeze Ltd and he was sponsored by GE 
Healthcare, Deep Breeze's distributor worldwide, to give lectures in academic meetings. YAG is an employee of Deep Breeze Ltd. and has stock options (no current monetary value).

\section{REFERENCES}

[1] Dosani R, Kraman SS. Lung sound intensity variability in normal men. A contour phonopneumographic study. Chest 1983; 83: 62831 .

[2] Kompis M, Pasterkamp H, Wodicka GR. Acoustic imaging of the human chest. Chest 2001; 120: 1309-21.

[3] Charleston-Villalobos S, Cortés-Rubiano S, González-Camarena R, Chi-Lem G, Aljama-Corrales T. Respiratory acoustic thoracic imaging (RATHI): assessing deterministic interpolation techniques. Med Biol Eng Comput 2004; 42: 618-26.

[4] Charleston-Villalobos S, González-Camarena R, Chi-Lem G, Aljama-Corrales T. Acoustic thoracic images for transmitted glottal sounds. Conf Proc IEEE Eng Med Biol Soc 2007; 2007: 3481-4.

[5] Dellinger PR, Parrillo JE, Kushnir A, Rossi M, Kushnir I. Dynamic visualization of lung sounds with a vibration response device: a case series. Respiration 2008; 75: 60-72.

[6] Dellinger RP, Jean S, Cinel I, Rajanala S, Glickman YA, Parillo JE. Regional distribution of acoustic-based lung vibration as a function of mechanical ventilation mode. Crit Care 2007; 11: R26.

[7] Lev S, Glickman YA, Kagan I, et al. Changes in regional distribution of lung sounds as a function of positive end-expiratory pressure. Crit Care 2009; 13: R66.

[8] Vena A, Perchiazzi, Giuliani R, Fiore T, Hedenstierna G. Acoustic effects of positive end-expiratory pressure on normal lung sounds in mechanically ventilated pigs. Clin Physiol Funct Imaging 2006; 26: $45-53$

[9] Lev S, Singer P, Glickman YA. Vibration response imaging: a novel technology for lung monitoring in critically ill patients. In: Esquinas A, Ed. Yearbook of Respiratory Care Clinics and Applied Technologies. Murcia, World Federation of Respiratory Care and Applied Technologies 2008; pp. 530-9.

[10] Ploy-Song-Sang Y, Martin RR, Ross WR, Loudon RG, Macklem PT. Breath sounds and regional ventilation. Am Rev Respir Dis 1977; 116: 187-99.

[11] Ploy-Song-Sang Y, Macklem PT, Ross WR. Distribution of regional ventilation measured by breath sounds. Am Rev Respir Dis 1978; 117: 657-64.

[12] Gavriely N, Cugell DW. Airflow effects on amplitude and spectral content of normal breath sounds. J Appl Physiol 1996; 80: 5-13.

[13] Leblanc P, Macklem PT, Ross RD. Breath sounds and distribution of pulmonary ventilation. Am Rev Respir Dis 1970; 102: 10-6.

[14] Kraman SS. The relationship between airflow and lung sound amplitude in normal subjects. Chest 1984; 86: 225-9.

[15] Hossain I, Moussavi Z. Relationship between airflow and normal lung sounds. Proc IEEE Canadian Conf Electrical Comput Eng 2002; 2: 1120-2

[16] Shykoff BE, Ploysongsang Y, Chang HK. Airflow and normal lung sounds. Am Rev Respir Dis 1988; 137: 872-6.

[17] Olson DE, Hammersley JR. Mechanisms of lung sound generation. Semin Respir Med 1985; 6: 171-9.

[18] Olson DE, Bogyi M, Schwartz DB, Hammersley JR. Relationship of tracheal breath sounds to airflow. Am Rev Respir Dis 1984; 129: A256.

[19] Charbonneau G, Sudraud M, Soufflet G. Method for the evaluation of flow rate from pulmonary sounds. Bull Eur Physiopathol Respir 1987; $23: 265-70$.
[20] Soufflet G, Charbonneau G, Polit M, et al. Interaction between tracheal sound and flow rate: a comparison of some different flow evaluations from lung sounds. IEEE Trans Biomed Eng 1990; 37 : 384-91.

[21] Harper VP, Pasterkamp H, Kiyokawa H, Wodicka GR. Modeling and measurement of flow effects on tracheal sounds. IEEE Trans Biomed Eng 2003; 50: 1-10.

[22] Mussell MJ, Miyamoto Y. Comparison of normal respiratory sounds recorded from the chest and trachea at various respiratory air flow levels. Front Med Biol Eng 1992; 4: 73-85.

[23] Yadollahi A, Moussavi ZM. Acoustical respiratory flow. A review of reliable methods for measuring air flow. IEEE Eng Med Biol Mag 2007; 26: 56-61.

[24] Nakano H, Sano K, Maekawa J, Narita N. Relationship between power spectra of breath sounds and inspiratory flows at different frequencies. Nippon Kyobu Shikkan Gakkai Zasshi 1994; 32: 1142-8.

[25] Betz A. Wind energy and its extraction through wind mills. Vieweg, Gottingen; 1926.

[26] Milic-Emili J, Henderson JA, Dolovich MB, Trop D, Kaneko K. Regional distribution of inspired gas in the lung. J Appl Physiol 1966; 21: 749-59.

[27] Robertson PC, Anthonisen NR, Ross D. Effect of inspiratory flow rate on regional distribution of inspired gas. J Appl Physiol 1969; 26: 438-43.

[28] Bake B, Wood L, Murphy B, Macklem PT, Milic-Emili J. Effect of inspiratory flow rate on regional distribution of inspired gas. J Appl Physiol 1974; 37: 8-17.

[29] Maher TM, Gat M, Allen D, Devaraj A, Wells AU, Geddes DM. Reproducibility of dynamically represented acoustic lung images from healthy individuals. Thorax 2008; 63: 542-8.

[30] Pasterkamp H, Patel S, Wodicka GR. Asymmetry of respiratory sounds and thoracic transmission. Med Biol Eng Comput 1997; 35: 103-6.

[31] O'Donnell DM, Kraman SS. Vesicular lung sound amplitude mapping by automated flow-gated phonopneumography. J Appl Physiol 1982; 53: 603-9.

[32] Gross V, Dittmar A, Penzel T, Schüttler F, von Wichert P. The relationship between normal lung sounds, age and gender. Am J Respir Crit Care Med 2000; 162: 905-9.

[33] Kramer MR, Raviv Y, Hardoff R, Shteinmatz A, Amital A, Shitrit D. Regional breath sound distribution analysis in single-lung transplant recipients. J Heart Lung Transplant 2007; 26: 1149-54.

[34] Mor R, Kushnir I, Meyer JJ, Ekstein J, Ben-Dov I. Breath sound distribution images of patients with pneumonia and pleural effusion. Respir Care 2007; 52: 1753-60.

[35] Anantham D, Herth FJ, Majid A, Michaud G, Ernst A. Vibration response imaging in the detection of pleural effusions: a feasibility study. Respiration 2009; 77: 166-72.

[36] Blanco M, Mor R, Fraticelli A, Breen DP, Dutau H. Distribution of breath sound images in patients with pneumothoraces compared to healthy subjects. Diagnostic yield of vibration response imaging technology. Respiration 2009; 77: 173-8.

[37] Jean S, Cinel I, Tay C, et al. Assessment of asymmetric lung disease in intensive care unit patients using vibration response imaging. Anesth Analg 2008; 107: 1243-7.

[38] Wang Z, Jean S, Bartter T. Lung sound analysis in the diagnosis of obstructive airway disease. Respiration 2009; 77: 134-8.

[39] Guntupalli KK, Alapat PM, Bandi VD, Kushnir I. Validation of automatic wheeze detection in patients with obstructed airways and in healthy subjects. J Asthma 2008; 45: 923-30.

[40] Guntupalli KK, Reddy RM, Loutfi RH, Alapat PM, Bandi VD, Hanania NA. Evaluation of obstructive lung disease with vibration response imaging. J Asthma 2008; 45: 903-7. 